$48 \mid 2017$

L'art de mentir

\title{
Abish Aynur, Modality in Kazakh as spoken in China
}

Wiesbaden, Harrassowitz Verlag, 2016, XIX-250 pages, 5 tableaux, 9 textes transcrits, glosés et traduits, ISBN 978-3-447-10626-9

\section{Camille Simon}

\section{(2) OpenEdition}

Journals

Édition électronique

URL : https://journals.openedition.org/emscat/2928

DOI : 10.4000/emscat.2928

ISSN : 2101-0013

Éditeur

Centre d'Etudes Mongoles \& Sibériennes / École Pratique des Hautes Études

\section{Référence électronique}

Camille Simon, "Abish Aynur, Modality in Kazakh as spoken in China », Études mongoles et sibériennes, centrasiatiques et tibétaines [En ligne], 48 | 2017, mis en ligne le 05 décembre 2017, consulté le 13 juillet 2021. URL : http://journals.openedition.org/emscat/2928; DOI : https://doi.org/10.4000/emscat.2928

Ce document a été généré automatiquement le 13 juillet 2021.

(c) Tous droits réservés 


\section{Abish Aynur, Modality in Kazakh as spoken in China}

Wiesbaden, Harrassowitz Verlag, 2016, XIX-250 pages, 5 tableaux, 9 textes transcrits, glosés et traduits, ISBN 978-3-447-10626-9

\section{Camille Simon}

\section{RÉFÉRENCE}

Abish Aynur, Modality in Kazakh as spoken in China, Wiesbaden, Harrassowitz Verlag, 2016

Cet ouvrage est issu de la thèse de doctorat de l'auteure, soutenue à l'université d'Uppsala en 2014, sous la direction d'É. Csató-Johanson. La première partie, de 164 pages, constitue l'étude proprement dite: celle-ci est suivie d'un long appendice (89 pages) qui contient le corpus exploité par l'auteure: neuf textes originaux, de longueur variable (4 à 77 énoncés). Les textes enregistrés, qui sont tous des monologues, sont transcrits, annotés et traduits (en anglais) par l'auteure. Enregistrés entre 2010 et 2012 dans la province du Xinjiang (Chine), auprès de locuteurs âgés de 30 à 72 ans, ils représentent la langue kazakhe contemporaine parlée en République Populaire de Chine. Ces monologues relèvent de plusieurs genres : discours explicatifs (T1, T5 \& T8) récits historiques et/ou biographiques ou autobiographiques (T2, T3 \& T4), instructions (T6 \& T7), récit d'anecdote/expérience personnelle (T9). Chaque texte est accompagné de métadonnées précises: sexe, âge, date, lieu de naissance et compétences linguistiques du locuteur, date et lieu d'enregistrement par l'auteure. Ces indications précises et systématiques ajoutent encore à la valeur documentaire de ce corpus original, composé de données naturelles. Il est cependant dommage que l'auteure ne propose pas de carte schématique de la région du Xinjiang, qui aiderait les lecteurs qui ne sont pas familiers de la région à localiser les zones habitées par des locuteurs de kazakh (et des autres langues de la région) ainsi que les lieux d'enregistrements. Ces données naturelles de première main sont complétées par des données textuelles (textes publiés au Kazakhstan et en Chine) et des données recueillies 
par élicitation en kazakh et en ouighour (sans plus de précision sur les méthodes employées dans ce cas).

2 Une première section introductive (10 pages) présente la situation sociolinguistique de la langue kazakhe en Chine et détaille les études publiées en kazakh et en chinois dans différentes institutions de la RPC. Dans le développement de l'étude, l'auteure cite et s'appuie régulièrement sur ces sources, en particulier celles publiées en chinois, ce qui sera particulièrement apprécié des lecteurs non-sinophones pour qui l'accès à ces sources est difficile.

3 L'auteure présente ensuite (en 7 p.) le cadre théorique développé par Johanson pour décrire les catégories de modalités dans les langues turciques, qu'elle appliquera pour décrire de façon détaillée ce domaine linguistique en kazakh. Le domaine de la modalité, présenté comme l'«attitude du locuteur" par rapport au contenu propositionnel, est un domaine particulièrement vaste, et dont la définition et les limites ne font pas l'objet d'un consensus (Palmer [1986] 2001; Nuyts 2006). Cette étude s'inscrit dans une perspective résolument descriptiviste et l'auteure ne discute donc pas la littérature typologique sur le sujet ni ne confronte le cadre proposé par Johanson à d'autres cadres descriptifs ou théoriques. Le cadre défini par Johanson distingue trois types de modalités : la volition (définie comme une attitude de désir ou de souhait visà-vis de la réalisation du contenu propositionnel), la nécessité (correspondant à la modalité déontique) et l'épistémique. Les marques de modalités illocutoires sont également prises en compte. Celles-ci sont définies comme des moyens, pour le locuteur, d'apporter une appréciation sur le contenu de ses propos («adresser's comment on his or her utterance» p. 16). L'auteure définit donc trois dimensions à son objet d'étude, qu'elle décrit séparément.

4 On peut regretter qu'elle ne discute pas des relations que ces différents types de modalité entretiennent entre eux dans la grammaire du kazakh : en effet, la description montre, en particulier, que les marqueurs de modalités illocutoires peuvent souvent se combiner avec des morphèmes marquant d'autres types de modalités (voir par exemple p. 107, sur la combinaison de la particule $\dot{a}$ avec les modes volontatif et impératif). Une analyse des combinaisons possibles entre les marqueurs des différents types de modalité en kazakh aurait été la bienvenue, afin de mieux comprendre l'étendue des différents domaines sémantiques qu'ils expriment et la manière dont ils s'articulent entre eux. Cet aspect de la description aurait également été utile aux typologues s'intéressant à la manière dont le domaine de la modalité est grammaticalisé et structuré dans les langues. Dans la suite de son étude, l'auteure propose une description très détaillée de chacun des marqueurs de modalité, si bien qu'une lecture attentive de l'ouvrage permet de reconstituer ces informations disséminées dans l'ensemble du texte. Cependant, une présentation synthétique de cette question faciliterait la lecture.

5 L'auteure détaille ensuite la distinction entre modalité subjective (attitude correspondant au point de vue du locuteur) et objective (attitude correspondant à un point de vue extérieur). Cette opposition sémantique correspond en effet, dans certains cas, à une opposition de procédés grammaticaux ou lexicaux spécifiques en kazakh et des exemples sont proposés au cas par cas. Ainsi, par exemple, tandis que kerek peut être employé pour exprimer indifféremment une modalité déontique subjective ou objective (p. 126-127), mäžbür n'est employé que pour exprimer une modalité déontique objective, et jamais subjective (p. 129). De même, la forme adverbiale árine exprime une 
modalité épistémique subjective lorsqu'elle est accentuée, mais objective lorsqu'elle est non-accentuée (p.120-121). Il aurait été intéressant de proposer une synthèse, par exemple sous forme de tableau récapitulatif en fin d'ouvrage, des cas où cette opposition est pertinente dans la grammaire de la langue, afin de mieux la mettre en valeur.

6 Finalement, l'auteure aborde les notions de « capacité » et d'« intention ». Alors que ces deux notions sont fréquemment incluses dans les descriptions de la modalité dans les langues, l'auteure considère qu'elles ne relèvent pas de ce domaine, dans la mesure où elles n'expriment pas, à proprement parler, une attitude du locuteur vis-à-vis du contenu propositionnel. L'expression grammaticale de la capacité et de l'intention en kazakh est néanmoins abordée de façon marginale dans le corps de l'étude et dans une section finale intitulée " Non-Modal Expressions » (12 p.).

7 Après cette courte introduction, la description proprement dite des données occupe l'essentiel de l'ouvrage. Il faut dire quelques mots de la manière dont les données sont présentées, ainsi que de la structuration générale de l'étude. L'auteure affirme choisir une perspective onomasiologique (" asking what devices Kazakh applies in order to express various semantic notions », p. 1), mais présente ensuite les procédés grammaticaux ainsi dégagés en fonction de leurs caractéristiques morphosyntaxiques. Ainsi le cœur de la description se structure en trois parties : une première partie $(51$ p.) est consacrée à l'expression des modalités à l'aide des modes verbaux, une seconde partie $(43 \mathrm{p}$.) s'intéresse aux particules modales et une troisième partie (37 p.), aux expressions lexicales et en particulier aux adverbes. Cependant, l'auteure oscille en réalité entre les deux approches, sémasiologique et onomasiologique. Ainsi, elle présente par exemple des usages non-modaux du mode hypothétique (p. 42-55 - certains exemples de cette section illustrent des fonctions modales, mais ce n'est pas le cas pour la plupart d'entre eux), ce qui correspond donc à une approche sémasiologique. À l'inverse, dans la section consacrée au mode impératif, elle présente une sous-partie intitulée "other means to express orders» (p.25) ce qui correspond, cette fois, à une approche plus strictement onomasiologique.

8 De tels changements de perspective se trouvent tout au long de l'ouvrage. Ils se justifient par l'objectif de proposer une description détaillée et complète des phénomènes liés à la modalité dans la langue, cependant, cette structuration ne facilite pas la lecture, surtout lorsqu'elle n'est pas clairement explicitée. La modalité, telle que définie dans le cadre théorique de Johanson, délimite un domaine fonctionnel d'ordre sémantico-pragmatique. L'adoption d'une perspective plus strictement onomasiologique et une structuration de l'ouvrage selon les quatre grandes souscatégories de modalité définies - volition; épistémique; déontique et illocutoire/ énonciative - nous aurait semblé préférable.

9 Il est vrai que, dans ce cas, certaines formes - comme le mode optatif qui exprime à la fois des valeurs de volition et d'épistémique (p.35) - apparaitraient dans plusieurs sections et c'est sans doute pour cette raison que l'auteure a choisi de présenter sa description en se basant sur les formes grammaticales ou lexicales, et non sur les notions. Ce choix présente également l'avantage, pour les lecteurs spécialistes d'autres langues turciques, d'effectuer facilement des comparaisons entre les formes communes des langues de la famille. Il en résulte cependant une multiplication des sous-catégories sémantico-pragmatiques de modalité pour chaque forme. Celles-ci semblent souvent définies de façon ad hoc pour chaque morphème ou pour chaque construction et on 
aboutit souvent à un certain foisonnement de notions. Ainsi, par exemple, les fonctions du mode volontatif sont définies de la façon suivante: "Voluntative markers express notions of will, desire, wishing, intention, request, command, demand, entreaty, advice, recommendation, exhortation, warning, hope, permission, or possibility with respect to the fulfillment of a given action » (p. 27). Ou encore, pour la description de la particule aw: "It takes the entire proposition within its scope and expresses the addresser's evaluation of its truth, including assumption, incredulity, surprise, etc. » (p. 87). Sauf dans de rares cas, l'auteure ne contraste pas explicitement les différentes formes qui expriment des notions proches, afin de mettre en évidence les différences et les similitudes fonctionnelles entre elles. Il ne s'agit pas de nier la complexité des fonctions assumées par les différents marqueurs de modalité en kazakh, mais pour le lecteur, l'abondance de détails et le manque de comparaisons et de synthèses rendent parfois difficile la compréhension du système modal dans sa globalité. Des comparaisons plus systématiques des fonctions exprimées par les différentes formes étudiées auraient permis de faire émerger les notions pertinentes pour décrire le système dans son ensemble. Il faut cependant souligner que les différentes fonctions énumérées sont illustrées par de nombreux exemples ce qui confère une valeur documentaire précieuse à cet ouvrage.

10 Pour conclure sur ce point, il convient d'ajouter que les deux types de présentation des données - onomasiologique ou sémasiologique - présentent chacune des avantages et des inconvénients propres. Le choix final procède des inclinations et des objectifs spécifiques de l'auteure et s'avère plus ou moins pertinent en fonction des attentes et de la perspective du lecteur. Une solution relativement simple aurait cependant permis de dépasser cette opposition et de répondre aux deux perspectives, sans pour autant remettre en question le choix de présentation fait par l'auteure. En effet, celle-ci présente trois tableaux récapitulatifs en conclusion de l'ouvrage (p. 156-157). Les deux premiers reprennent la présentation sémasiologique de l'ouvrage: ils récapitulent respectivement les fonctions modales des modes et des particules modales. Le dernier classe les expressions lexicales en fonction de trois grands types de modalité (volition, nécessité et possibilité). Il aurait été intéressant que l'auteure propose, dans cette conclusion, une double série de tableaux récapitulatifs: une série se basant sur les formes pour indiquer les notions qu'elles véhiculent, et une série partant des notions, et énumérant les formes - grammaticales, lexicales et constructions analytiques employées pour exprimer ces notions. Ce type de synthèses aurait facilité la lecture et la compréhension des données.

11 Malgré ces remarques, il faut encore une fois souligner que l'insuffisance de synthèses récapitulatives n'est que l'aspect secondaire d'une description particulièrement détaillée et approfondie proposée par l'auteure. Il importe en effet de préciser que chaque forme exprimant une notion modale est décrite dans ses multiples dimensions. Ainsi, les différentes catégories d'expressions sont bien définies formellement: par exemple, les modes sont définis comme des "synthetic devices consisting of bound inflectional suffixes and expressing different basic modal notions » (p. 18). De la même façon, les particules modales sont clairement distinguées des adverbes par leurs propriétés morphosyntaxiques et l'auteure fournit des indications prosodiques; pour les formes grammaticales, l'auteure ne manque pas de préciser leur caractère productif ou non en synchronie; d'une manière générale, elle décrit systématiquement les phénomènes d'allomorphie, propose des hypothèses quand à l'origine étymologique des formes. Notons également que les comparaisons très fréquentes avec les langues de la famille et 
les langues en contact sont particulièrement bienvenues et donnent à l'étude une dimension génétique et aréale. La description ne manque pas non plus de présenter et de décrire systématiquement les formes analytiques complexes construites à l'aide d'un suffixe de mode, d'une particule ou d'un adverbe modal.

Comme on l'a mentionné plus haut, cela amène parfois l'auteure à s'éloigner de la question principale de son étude, pour décrire des fonctions non-modales de certaines de ces constructions. L'auteure semble avoir inclus toutes les formes rencontrées dans son corpus et dans les descriptions et la bibliographie consultée, mais elle ne définit pas explicitement les critères d'inclusion des formes. Ainsi, elle ne donne pas d'information sur le degré de grammaticalisation et de spécialisation des formes traitées : la réponse à cette question reste implicite, la grammaticalisation et la spécialisation dépendant du type morphosyntaxique de la forme considérée. Des détails sur le degré de grammaticalisation des différentes formes lexicales auraient pu être donnés pour préciser encore la description.

13 Enfin, il faut signaler que la description comporte de très nombreux exemples (462 exemples glosés et traduits au total) qui illustrent chacune des fonctions, modales ou non, des formes décrites dans l'ouvrage, et chacune des constructions mises en évidence. Sur cet ensemble, on peut regretter que seuls 36 exemples soient issus du corpus naturel recueilli par l'auteure. La plupart des exemples cités sont en effet des exemples élicités, tandis qu'un plus petit nombre provient des références bibliographiques, et notamment des travaux publiés en langue chinoise (et qui ne sont pas aisément accessible à tous les linguistes travaillant sur les langues turciques).

14 Pour conclure, cet ouvrage, qui présente une analyse très détaillée et précise des formes exprimant des valeurs modales en kazakh, est une contribution importante à l'étude de la modalité dans les langues turciques. Les quelques nuances, tenant à un manque de synthèses récapitulatives, ne doivent pas éclipser la valeur descriptive, particulièrement appréciable, de cette étude, qui en fait une source précieuse pour l'étude des systèmes de modalité dans les langues.

\section{BIBLIOGRAPHIE}

Nuyts, J. 2006 Modality. Overview and linguistic issues, in W. Frawley (éd.) The expression of modality (Berlin, Mouton de Gruyter), pp. 1-26.

Palmer, F. R. [1986] 2001 Mood and Modality (Cambridge, Cambridge University Press).

\section{AUTEURS}

\section{CAMILLE SIMON}

ATER à l'Université d'Aix-Marseille, affiliée aux laboratoires LPL (UMR 6057) et LACITO (UMR 7107) 